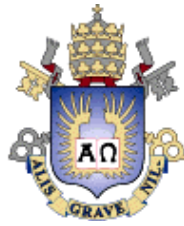

Eduardo Magno Lages Figueiredo

\title{
Uma Abordagem Quantitativa para Desenvolvimento de Software Orientado a Aspectos
}

Dissertação de Mestrado

Dissertação apresentada como requisito parcial para obtenção do título de Mestre pelo Programa de Pós-Graduação em Informática da PUC-Rio.

Orientadores: Carlos José Pereira de Lucena Alessandro Fabricio Garcia 
Eduardo Magno Lages Figueiredo

\title{
Uma Abordagem Quantitativa para Desenvolvimento de Software Orientado a Aspectos
}

\begin{abstract}
Dissertação apresentada como requisito parcial para obtenção do título de Mestre pelo Programa de PósGraduação em Informática da PUC-Rio. Aprovada pela Comissão Examinadora abaixo assinada.
\end{abstract}

Prof. Carlos José Pereira de Lucena Orientador PUC-Rio

Prof. Alessandro Fabricio Garcia Co-Orientador Universidade de Lancaster - UK

Prof. Arndt von Staa PUC-Rio

Prof. Renato Fontoura de Gusmão Cerqueira PUC-Rio

Prof. José Eugenio Leal Coordenador Setorial do Centro Técnico Científico - PUC-Rio

Rio de Janeiro, 29 de março de 2006 
Todos os direitos reservados. É proibida a reprodução total ou parcial do trabalho sem autorização da universidade, do autor e dos orientadores.

\section{Eduardo Magno Lages Figueiredo}

Graduou-se em Ciência da Computação na Universidade Federal de Ouro Preto (UFOP) em 2003.

Ficha Catalográfica

Figueiredo, Eduardo Magno Lages

Uma abordagem quantitativa para desenvolvimento de software orientado a aspectos / Eduardo Magno Lages Figueiredo ; orientador: Carlos José Pereira de Lucena; co-orientador: Alessandro Fabricio Garcia. - Rio de Janeiro : PUC-Rio, Departamento de Informática, 2006.

140 f. ; $30 \mathrm{~cm}$

Dissertação (mestrado) - Pontifícia Universidade Católica do Rio de Janeiro, Departamento de Informática.

Inclui referências bibliográficas.

1. Informática - Teses. 2. Desenvolvimento de software orientado a aspectos. 3. Método de avaliação. 4. Engenharia de software experimental. 5. Software. 6. Padrões de projeto. I. Lucena, Carlos José Pereira de. II. Garcia, Alessandro Fabrício Garcia. III. Pontifícia Universidade Católica do Rio de Janeiro. Departamento de Informática. IV. Título. 


\section{Agradecimentos}

Em primeiro lugar, gostaria de agradecer aos meus orientadores Carlos Lucena e Alessandro Garcia, pois sem eles este trabalho não teria sido possível. O professor Lucena trouxe apoio, confiança, ensinamentos e liberdade para escolher o trabalho, enquanto o amigo Alessandro complementou com incentivo, criatividade, dinamismo e adrenalina (nos deadlines).

Aos professores do Departamento de Informática que contribuíram para a minha formação. Em especial, ao professor Arndt von Staa por ter me acompanhado desde o meu primeiro relatório técnico pelo mundo de medições e qualidade de software. Também ao professor Renato Cerqueira pelas discussões que permitiram melhorias neste documento.

Aos participantes dos estudos experimentais que contribuíram diretamente para os resultados alcançados: Alessandro Garcia, Cláudio Sant'Anna, Carlos Lucena, Uirá Kulesza, Arndt von Staa, Nélio Cacho, Thaís Batista, Fernando Castor, Cecília Rubira e Thiago Bartolomei. Em particular, ao amigo Cláudio Sant'Anna que foi fundamental para os caminhos traçados e compartilhou cada idéia desta dissertação.

Agradeço também ao professor Marcelo Maia (UFOP) pelos primeiros passos na Engenharia de Software e pelas conversas sobre manipulação de programas.

Aos colegas do Laboratório de Engenharia de Software, onde encontrei um espaço privilegiado para desenvolvimento de meu trabalho, em especial ao grupo “Aspectos@PUC-Rio” pelas frutíferas discussões.

À Vera Menezes pelo carinho e apoio, especialmente em questões administrativas. À CAPES, à PUC-Rio e à Fundação Padre Leonel Franca, pelo apoio financeiro. Agradeço aos meus pais José e Sônia, aos meus irmãos Ângelo, Rosane e Marcelo e à minha princesinha Marina, pelo incentivo, carinho e amor incondicional.

À Deus, por me agraciar com tantas conquistas. 


\section{Resumo}

Figueiredo, Eduardo. Uma Abordagem Quantitativa para Desenvolvimento de Software Orientado a Aspectos. Rio de Janeiro, 2006. 140p. Dissertação de Mestrado - Departamento de Informática, Pontifícia Universidade Católica do Rio de Janeiro.

O desenvolvimento de software orientado a aspectos é um paradigma recente que introduz novas abstrações e mecanismos com o objetivo de melhorar a modularidade de interesses que se espalham pelo sistema. Entretanto, a satisfação de atributos de qualidade em sistemas orientados a aspectos não é tarefa simples e a utilização equivocada destas novas abstrações pode resultar em efeitos colaterais relacionados a princípios importantes da Engenharia de Software, tais como elevado acoplamento, baixa coesão dos módulos e incompleta modularidade dos interesses em aspectos. Problemas como estes não são facilmente verificáveis em sistemas de médio e grande porte sem um método adequado e, geralmente, consomem muito tempo e recursos. Portanto, torna-se necessário um método de avaliação que auxilie engenheiros de software na análise de sistemas orientados a aspectos. Este trabalho de mestrado propõe uma abordagem que provê suporte à avaliação quantitativa de implementações orientadas a aspectos. A abordagem incluiu: (i) um método de avaliação organizado em etapas, e (ii) uma ferramenta de medição e avaliação, chamada AJATO, que dá suporte ao método proposto. O método é composto por um conjunto de métricas e regras heurísticas. As métricas fornecem informações quantitativas e as heurísticas contribuem com algum raciocínio semântico dos números. A ferramenta AJATO é composta por quatro módulos que efetuam o parser do código, mapeamento de estruturas sintáticas em interesses, medição e avaliação heurística. Um conjunto de cinco estudos de caso envolvendo domínios de aplicação distintos foi realizado para avaliar a utilidade e usabilidade da abordagem proposta.

\section{Palavras-chave}

Desenvolvimento de software orientado a aspectos, método de avaliação, métricas de software, engenharia de software experimental, padrões de projeto. 


\section{Abstract}

Figueiredo, Eduardo. A Quantitative Approach to Aspect Oriented Software Development. Rio de Janeiro, 2006. 140p. Master Thesis Computer Science Department, Pontifical Catholical University of Rio de Janeiro.

Aspect-oriented software development is an emerging paradigm that provides new abstractions and mechanisms to support the modularization of crosscutting concerns through the software development lifecycle. However, the achievement of high-quality aspect-oriented software is not trivial. The inappropriate use of aspect-oriented abstractions and mechanisms potentially leads to the violation of important design principles, such as low coupling, high cohesion, incomplete modularization of crosscutting concerns into aspects, and so forth. These problems are not easily detectable and an ad hoc analysis of large designs and implementations is often expensive and time-consuming. Hence there is a need for an assessment method that assists software engineers in the analysis of their aspect-oriented implementations. This work proposes the development of a systematic approach to support the quantitative assessment of aspect-oriented software. The approach is organized in a stepwise fashion and is founded on a metrics suite and a comprehensive set of complementary rules. Our proposal is supported by a measurement and assessment tool. A set of five case studies from different application domains have been carried out in order to evaluate the usability and usefulness of our proposed approach.

\section{Keywords}

Aspect-Oriented Software Development, Assessment Method, Software Metrics, Empirical Software Engineering, Design Patterns. 


\section{Sumário}

1 Introdução 15

1.1. Definição do Problema 15

1.2. Limitações dos Trabalhos Relacionados 16

1.3. Solução Proposta 17

1.4. Organização do Texto 18

2 Desenvolvimento de Software Orientado a Aspectos 20

2.1. AspectJ: uma Extensão de Java para Orientação a Aspectos 22

2.2. Alguns Exemplos de Utilização de Aspectos 26

3 Estado da Arte e Trabalhos Relacionados 29

3.1. Qualidade de Software 29

3.2. Métricas de Software $\quad 30$

3.3. Avaliação de Software Baseado em Métricas 35

3.4. Ferramentas de Desenvolvimento Orientado a Aspectos 37

3.5. Limitações dos Trabalhos Relacionados 40

4 O Método de Avaliação 42

4.1. Artefatos e Recursos do Método 44

4.2. Atividades do Método 50

5 Regras Heurísticas $\quad 56$

5.1. Problemas de Interpretação em Medições 57

5.2. Regras Heurísticas de Separação de Interesses 64

5.3. Regras Heurísticas de Acoplamento e Coesão 70

5.4. Definição de Valores Limite 74

6 A Ferramenta de Medição e Avaliação 77

$\begin{array}{ll}\text { 6.1. Modelo Arquitetural } & 78\end{array}$ 
7 Estudos Experimentais $\quad 92$

7.1. Sistemas Utilizados nos Estudos Experimentais 92

7.2. Contribuições dos Estudos 100

7.3. Restrições dos Estudos 102

8 Considerações Finais 104

8.1. Contribuições 104

8.2. Trabalhos Futuros 106

9 Referências 109

Apêndice A Resultados Estudo Experimental dos Padrões 115

Apêndice B Resultados do Estudo Experimental Middleware OpenOrb122

Apêndice C Resultados do Estudo Experimental Portalware 132

Apêndice D Resultados do Estudo Experimental Health Watcher 136 


\section{Lista de figuras}

Figura 1 - Separação de interesses (a) bidimensional e (b) tridimensional 21

Figura 2 - Interesse transversal em sistemas (a) OO e (b) OA 22

Figura 3 - Exemplo de aspecto para tratamento de falhas 26

Figura 4 - Exemplo de aspecto para associação de papel a classes 27

Figura 5 - Exemplo de aspecto para registrar tempo de conexão com servidor 28

Figura 6 - Diagrama de classes OO do editor de figuras 33

Figura 7 - Sombreamento da classe Line do editor de figuras 34

Figura 8 - Modelo de qualidade proposto por Sant'Anna et al. [57] 36

Figura 9 - Atividades do Concern Manipulation Environment 37

Figura 10 - Módulos da ferramenta de Ceccato e Tonella [13] 40

Figura 11 - Método progressivo para avaliação de software 43

Figura 12 - Diagrama de classes do padrão Mediator 46

Figura 13 - Sombreamento da classe Button do padrão Mediator 48

Figura 14 - Diagrama de classes OO do padrão Prototype 54

Figura 15 - Diagrama de classes OA do padrão Prototype 54

Figura 16 - Diagrama de classes OO destacando Observer e Factory Method 58

Figura 17 - Diagrama de classe OO destacando padrões Façade e Singleton $\quad 61$

Figura 18 - Diagrama de estados dos interesses e regras de transição 65

Figura 19 - Diagrama de estados dos componentes e regras de transição 71

Figura 20 - Representação arquitetural da ferramenta AJATO 78

Figura 21 - Interface da ferramenta de avaliação $\quad 81$

Figura 22 - Janelas para carregar um sistema e respectiva ajuda $\quad 82$

Figura 23 - Estrutura de diretórios da ferramenta AJATO 83

Figura 24 - Modelo de decomposição de sistemas AspectJ 84

Figura 25 - Diagrama de classes parcial do Modelo AspectJ. 85

Figura 26 - Diagrama de classes parcial do módulo Extrator de Modelo AspectJ86

Figura 27 - Diagrama de classes parcial do módulo Gerenciador de Interesses 87

Figura 28 - Diagrama de classes parcial do módulo Coletor de Métricas 89

Figura 29 - Diagrama de classes da estrutura de medição 89 
Figura 30 - Diagrama de classes parcial do módulo Analisador de Regras 91

Figura 31 - Diagrama de classes OO parcial da middleware OpenOrb 95

Figura 32 - Diagrama de classes OO parcial do Portalware 97

Figura 33 - Diagrama de classes OA parcial do Portalware 97

Figura 34 - Diagrama de classes OO parcial do Health Watcher 99

Figura 35 - Diagrama de classes OA parcial do Health Watcher 99 


\section{Lista de tabelas}

Tabela 1 - Conjunto de métricas orientadas a aspectos do método 51

Tabela 2 - Conjunto de regras heurísticas baseadas em uma única métrica 52

Tabela 3 - Resultado das métricas de SI para Observer e Factory Method 59

Tabela 4 - Resultado de acoplamento e coesão para o padrão Factory Method 60

Tabela 5 - Resultado das métricas de SI para padrões Façade e Singleton 61

Tabela 6 - Resultado das métricas para o papel Subject do padrão Observer 64

Tabela 7 - Regras heurísticas de separação de interesses $\quad 67$

Tabela 8 - Regras heurísticas de acoplamento e coesão 73

Tabela 9 - Valores limite utilizados nas regras heurísticas 75

Tabela 10 - Elementos da abordagem avaliados nos estudos experimentais 93

Tabela 11 - Problemas identificados pelas regras heurísticas nos sistemas 101

Tabela 12 - Resultados de SI e tamanho para o papel Subject 115

Tabela 13 - Resultados de SI e tamanho para o papel Observer 115

Tabela 14 - Resultado de acoplamento e coesão para o padrão Observer 116

Tabela 15 - Regras de SI para o papel Subject do padrão Observer 116

Tabela 16 - Regras de SI para o papel Observer do padrão Observer 116

Tabela 17 - Regras de Acoplamento e Coesão para o padrão Observer 117

Tabela 18 - Resultados de SI e tamanho para o papel Creator 118

Tabela 19 - Resultado de acoplamento e coesão para o Factory Method 118

Tabela 20 - Regras de SI para o papel Creator do padrão Factory Method 118

Tabela 21 - Resultados de SI e tamanho para o papel Director 120

Tabela 22 - Resultado de acoplamento e coesão para o padrão Builder $\quad 120$

Tabela 23 - Regras de SI para o papel Director do padrão Builder 120

Tabela 24 - Resultados de SI e tamanho para o padrão Observer 122

Tabela 25 - Resultados de SI e tamanho para o padrão Factory Method 122

Tabela 26 - Resultado de acoplamento e coesão para Observer com Factory $\begin{array}{ll}\text { Method } & 123\end{array}$

Tabela 27 - Regras de SI para o padrão Observer 123

Tabela 28 - Regras de SI para o padrão Factory Method 123 
Tabela 29 - Regras de acoplamento e coesão para composição Observer com Factory Method 124

Tabela 30 - Resultados de SI e tamanho para o padrão Singleton 125

Tabela 31 - Resultados de SI e tamanho para o padrão Façade 125

Tabela 32 - Resultado de acoplamento e coesão para Singleton com Façade 125

Tabela 33 - Regras de SI para o padrão Singleton na composição com Façade 125

Tabela 34 - Regras de SI para o padrão Façade na composição com Singleton 126

Tabela 35 - Regras de acoplamento e coesão para Singleton com Façade 126

Tabela 36 - Resultados de SI e tamanho para o padrão Proxy 127

Tabela 37 - Resultados de SI e tamanho para o padrão Interpreter 127

Tabela 38 - Resultado de acoplamento e coesão para Proxy com Interpreter 128

Tabela 39 - Regras de SI para o padrão Proxy na composição com Interpreter 128

Tabela 40 - Regras de SI para o padrão Interpreter na composição com Proxy 128

Tabela 41 - Regras de acoplamento e coesão para Proxy com Interpreter 129

Tabela 42 - Resultados de SI e tamanho para o padrão State 130

Tabela 43 - Resultado de acoplamento e coesão para Prototype com State $\quad 130$

Tabela 44 - Regras de SI para o padrão State na composição com Prototype 130

Tabela 45 - Resultados de SI e tamanho para o interesse Adaptação 132

Tabela 46 - Resultados de SI e tamanho para o interesse Colaboração 132

Tabela 47 - Resultados de SI e tamanho para o interesse Autonomia 132

Tabela 48 - Resultado de acoplamento e coesão para o Portalware 133

Tabela 49 - Regras de SI para o interesse Adaptação 134

Tabela 50 - Regras de SI para o interesse Colaboração 134

Tabela 51 - Regras de SI para o interesse Autonomia 134

Tabela 52 - Regras de acoplamento e coesão para o Portalware 135

Tabela 53 - Resultados de SI e tamanho para o interesse Concorrência 136

Tabela 54 - Resultados de SI e tamanho para o interesse Distribuição 136

Tabela 55 - Resultado de acoplamento e coesão para o Health Watcher 137

Tabela 56 - Regras de SI para o interesse Concorrência do Health Watcher 139

Tabela 57 - Regras de SI para o interesse Distribuição do Health Watcher 139

Tabela 58 - Regras de acoplamento e coesão para o Health Watcher 139 


\section{Lista de métricas}

\begin{tabular}{|c|c|c|}
\hline Sigla & Nomes (Inglês e Tradução) & Referências \\
\hline CBC & $\begin{array}{l}\text { Coupling Between Components } \\
\text { Acoplamento entre Componentes }\end{array}$ & {$[31][57]$} \\
\hline CBO & $\begin{array}{l}\text { Coupling Between Objects } \\
\text { Acoplamento entre Objetos }\end{array}$ & [14] \\
\hline CDC & $\begin{array}{l}\text { Concern Diffusion over Components } \\
\text { Difusão de Interesse em Componentes }\end{array}$ & [31] [57] \\
\hline CDLOC & $\begin{array}{l}\text { Concern Diffusions over Lines of Code } \\
\text { Difusão de Interesse em Linhas de Código }\end{array}$ & {$[31][57]$} \\
\hline CDO & $\begin{array}{l}\text { Concern Diffusion over Operations } \\
\text { Difusão de Interesse em Operações }\end{array}$ & {$[31][57]$} \\
\hline DIT & $\begin{array}{l}\text { Depth Inheritance Tree } \\
\text { Profundidade da Árvore de Herança }\end{array}$ & {$[13][14]$} \\
\hline LCOO & $\begin{array}{l}\text { Lack of Cohesion in Operations } \\
\text { Perda de Coesão em Operações }\end{array}$ & [13] [57] \\
\hline LCOM & $\begin{array}{l}\text { Lack of Cohesion in Methods } \\
\text { Perda de Coesão em Métodos }\end{array}$ & [14] \\
\hline LOC & $\begin{array}{l}\text { Lines of Code } \\
\text { Número de Linhas de Código }\end{array}$ & {$[20][47]$} \\
\hline NOA & $\begin{array}{l}\text { Number of Attributes } \\
\text { Número de Atributos }\end{array}$ & {$[20][57]$} \\
\hline NOC & $\begin{array}{l}\text { Number of Children } \\
\text { Número de Filhos }\end{array}$ & [14] [22] \\
\hline NOO & $\begin{array}{l}\text { Number of Operations } \\
\text { Número de Operações }\end{array}$ & [20] [22] \\
\hline NOS & $\begin{array}{l}\text { Number of Statements } \\
\text { Número de Comandos }\end{array}$ & {$[20][22]$} \\
\hline
\end{tabular}




$\begin{array}{lll}\text { VS } & \text { Vocabulary Size } & \text { [31] [57] } \\ \text { WOC } & \text { Tamanho do Vocabulário } & \\ & \text { Weighted Operations per Component } & \text { [31] [57] } \\ \text { Peso das Operações por Componente } & \\ \text { WMC } & \text { Weighted Methods per Class } & \text { [13] [14] } \\ & \text { Peso dos Métodos por Classe } & \\ \text { NOAconcern } & \text { Number of Attributes per Concern } & \\ & \text { Número de Atributos do Interesse } & - \\ \text { NOOconcern } & \text { Number of Operations per Concern } & \\ & \text { Número de Operações do Interesse } & \\ & \text { Number of Lines of Code per Concern } & - \\ \text { LOCconcern } & \text { Número de Linhas de Código do Interesse } & \end{array}$

Neste documento os nomes das métricas são usados em português, entretanto, as suas respectivas siglas fazem referência aos nomes originais. 